\title{
Keypoint Descriptors in SIFT and SURF for Face Feature Extractions
}

\author{
SukTing Pui ${ }^{1}$ and Jacey-Lynn Minoi ${ }^{2}$ \\ University Malaysia Sarawak, Faculty of Computer Science and Information Technology, \\ 94300 Kota Samarahan, Sarawak, Malaysia. \\ ${ }^{1}$ polly_sukting@hotmail.com \\ ${ }^{2}$ jaceyefit.unimas.my
}

\begin{abstract}
The last decade, numerous researches are still working on developing a robust and faster keypoints image descriptors algorithm. In this paper, we will review a few complex keypoint descriptor approaches that are well-known and commonly used in vision applications, and they are Scale Invariant Feature Transform (SIFT) and Speed-up Robust Features (SURF). These methods aim to make the descriptors faster to compute and robust to scale, rotation and noise. We will the results of the experiments on face image data. The extracted keypoints and the regions of interest are analysed and compared against the corresponding facial features. The results have shown SIFT outperformed SURF in terms of speed while the extracted keypoints using SURF descriptors are mainly located on corners and distinct facial features.
\end{abstract}

Keywords: Keypoint Descriptors, SIFT, SURF, feature extraction.

\section{Introduction}

Over the past decade, applications in computer vision have dramatically increased and feature extraction is still at the base of many of these applications' problem. Earlier works of research have been focusing on extracting keypoint descriptors for better accuracy and improved speed.

Detecting facial features is a vital step in face recognition for image registration purposes. The accuracy of the feature extraction process is often influenced by face variants due to the changes in orientation, head poses, illumination, facial expressions, occlusion, cluttered background and so on. A number of methods have been studied and improved feature extraction. The commonly used feature extraction approaches are the Scale Invariant Feature Transform (SIFT) [1] and the Speeded Up Robust Features (SURF) [2]. These are known to be the most promising feature extraction methods for its high performance in various applications. Therefore, we will discuss further on the performance of these methods on face feature extraction purposes.

We have conducted a number of experiments on the existing 2D face images. The keypoint descriptors and the computational speed of both methods are compared. 